\title{
NOTE SUR LES RELATIONS DES VECTEURS DE LEISHMANIOSE AVEC LES ESSENCES FORESTIËRES EN GUYANE FRA NÇAISE
}

\author{
B. GEOFFROY*, J. P. DEDET**, J. LEBBE**, P. ESTERRE**, J. F. TRAPE*
}

\begin{abstract}
RÉSUMÉ. L'étude des relations entre phlébotomes et essences végétales a été entreprise en forêt guyanaise. Certaines essences semblent abriter plus particulièrement Lutzomyia umbratilis, vecteur de Leishmania braziliensis guyanensis qui représente pour chaque essence de 28 à $88 \%$ des captures sur troncs. L'association Lu. umbratilis + Lutzomyia rorotaensis en constitue de 58 à $98 \%$. Lutzomyia flaviscutellata, espèce vectrice de Leishmania mexicana amazonensis, capturé fréquemment sur homme, n'a pas été retrouvé sur tronc.
\end{abstract}

\section{Note on the relations between leishmaniasis vectors and forest trees in French Guiana.}

SUMMARY. The study of relationships between Phlebotomine sandflies and diflerent species of trees, 0 to $1,5 \mathrm{~m}$ above ground, was carried out in the French Guiana forest. We found that certain forest trees are more propitious than others to the development or maintenance of certain leishmaniasis vectors. Lu. umbratilis, vector of Leishmania braziliensis guyanensis, represents between 28 and $88 \%$ of samples collected on trunks. The association of $L u$. umbratilis and Lu. rorotaensis represents between 58 and $98 \%$. Lu. flaviscutellata, vector of Leishmania mexicana amazonensis, frequently caught on humans, was not found on tree trunks.

\section{1 - Introduction}

Les études menées depuis une dizaine d'années sur la transmission de la leishmaniose tégumentaire guyanaise ont montré que le cycle de Leishmania braziliensis guyanensis avec Lutzomyia umbratilis Ward et Fraiha, 1977 pour vecteur et Choloepus didactylus (Linné, 1758) pour réservoir, se déroulait au niveau de la voûte forestière (Lepont et Pajot, 1980).

Toutefois, Lu. umbratilis est capturé au niveau du sol, sur homme ou sur tronc d'arbre, pratiquement tout au long de l'année. Au sol se développe également un deuxième cycle (Dedet et al., 1985a) à Leishmania mexicana amazonensis Lainson

* Entomologistes médicaux de l'ORSTOM, 97306 Cayenne Cedex.

* Institut Pasteur de la Guyane Française, 97306 Cayenne Cedex, Guyane Française.

Accepté le rg janvier ı 986. 
et Shaw, 1972 évoluant entre Proechimys cuvieri Petter, 1978 et Lutzomyia flaviscutellata (Mangabeira, 1942). Il a cependant été montré que la majorité des cas humains de leishmaniose en Guyane française était due à L. b. guyanensis (Dedet et al., 1985b).

La recherche d'un lien entre végétation et phlébotomes pouvant favoriser une espèce vectrice, nous a incité à aborder le problème des biotopes naturels et leurs relations avec les différentes espèces.

Les troncs d'arbres font la transition entre la canopée où le cycle de $L . b$. guyanensis se développe et le sol où la contamination humaine se produit le plus souvent. Ils hébergent une population importante de phlébotomes et, qu'ils soient ou non associés aux biotopes de reproduction, ils représentent certainement un biotope particulier comme lieu de repos ou d'attente d'un repas.

Leur exploration jusqu'à 1,5 m du sol a été entreprise afin d'analyser le spectre des espèces présentes en fonction des différentes essences végétales et ainsi de mieux apprécier l'influence de la végétation sur les différentes composantes des populations vectrices : densité, répartition, compétition inter-spécifique, lieu de repos et de reproduction.

\section{2 - Matériel et méthode}

Cette recherche a été menée de février 1984 à mars 1985 dans deux stations implantées en forêt dense.

La station de F.R.G. $\left(52^{\circ} 35^{\prime} 0 / 4055^{\prime} \mathrm{N}\right)$ se situe sur le périmètre d'une exploitation forestière et le prélèvement de certaines essences ainsi que l'ouverture de pistes ont contribué à la secondarisation partielle de sa forêt.

La station de Nancibo $\left(52028^{\prime} 0 / 4040^{\prime} \mathrm{N}\right)$, plus au sud, offre une forêt moins perturbée mais avec pour particularité un sommet doléritique et un bas-fond inondé.

L'étude porte sur 30 arbres préalablement choisis (15 dans chaque station). Ils représentent 18 essences végétales, déterminées grâce à la collaboration de l'Office National des Forêts (tableau I) et correspondent tous à des espèces de forêt primaire.

Quatre essences sont communes aux deux sites : le Bois violet (Peltogyne sp.), l'Angélique (Dicorynia guianensis), le Balata franc (Manilkara bidentata) et le Bougou-bougou (Swartzia remiger). Elles représentent la moitié des arbres marqués des deux sites, soit 7 à F.R.G. et 8 à Nancibo.

Les phlébotomes ont été récoltés sur les troncs entre 0 et 1,5 mètre, de jour, par aspirateur à bouche, au cours de deux séances hebdomadaires dans les deux stations (soit un total de troncs visités de 450 à Nancibo et 390 à F.R.G.).

Parallèlement, des captures sur troncs non déterminés ont été pratiquées pour étendre notre recherche systématique des phlébotomes forestiers $(210$ troncs visités à F.R.G. et 90 à Nancibo).

Les phlébotomes ont été déterminés après éclaircissement, coloration et montage à l'Euparal ou après dissection. 
TABLEAU I. - Liste des essences végétales déterminées.

\begin{tabular}{|c|c|c|c|}
\hline Nom commun & Nom scientifique & F.R.G. & Nancibo \\
\hline 1 Bois violet & Peltogyne sp. (Césalpiniacées) & 2 & 4 \\
\hline 2 Angélique & Dicorynia guianensis (Césalpiniacées) & 1 & 2 \\
\hline 3 Wacapou & Vouacapoua americana (Césalpiniacées) & 1 & 0 \\
\hline 4 Taapoutiki & Dendrobangia boilviana (Icacinacées) & 1 & 0 \\
\hline 5 Balata franc & Manilkara bidentata (Sapotacées) & 2 & 1 \\
\hline 6 Niamboka & Pouteria guianensis (Sapotacées) & l & 0 \\
\hline $7 \begin{array}{l}\text { Bougou-bougou } \\
\text { (Bois corbeau) }\end{array}$ & Swartzia remiger (Césalpiniacées) & 2 & $\mathrm{I}$ \\
\hline 8 Tobitoutou & Sheffleva paraensis (Araliacées) & 1 & 0 \\
\hline 9 Balata pomme & Ecclinusa sanguinolenta (Sapotacées) & 1 & 0 \\
\hline 10 Bois jacquot & Laetia procera (Flacourtiacées) & $i$ & 0 \\
\hline 11 Anangossi & Terminalia amazonica (Combrétacées) & $\mathrm{i}$ & 0 \\
\hline 12 Kapokier & Bombax nervosum (Bombacacées) & 1 & 0 \\
\hline 13 Dodomissinga & Parkia nitida (Mimosacées) & 0 & 2 \\
\hline 14 Gonfolo-Kouali & Qualea sp. (Vochysiacées) & 0 & 1 \\
\hline 15 Chawari & Caryocar glabrum (Caryocariacées) & 0 & 1 \\
\hline 16 Akoinsiba & Pouteria sp. (Sapotacées) & 0 & 1 \\
\hline 17 Kopi & Goupia glabra (Célastracées) & 0 & 1 \\
\hline 18 Bioudou & Eperua falcata (Césalpiniacées) & 0 & 1 \\
\hline+ Bois violet & Peltogyne sp. (Césalpiniacées) & & \\
\hline
\end{tabular}

\section{3 - Résultats et commentaires}

\subsection{Résultats globaux}

4467 phlébotomes (3068 mâles et 1399 femelles), se rapportant à 21 espèces, ont été capturés sur 1140 troncs visités dans les deux stations (tableaux II et III).

$81,6 \%$ des phlébotomes ont été capturés à F.R.G. dont la forêt semble beaucoup plus propice au développement des phlébotomes.

Tableau II. - Effectifs des phlébotomes capturés sur troncs.

\begin{tabular}{crrr}
\hline & Nancibo & F.R.G. & Total \\
\hline $\begin{array}{l}\text { Nombre de phlébotomes sur troncs } \\
\text { déterminés } \\
\text { mâles } \\
\text { femelles }\end{array}$ & & \\
Total & 441 & 932 & 1373 \\
\cline { 2 - 4 } Nombre de phlébotomes sur troncs & 255 & 413 & 668 \\
indéterminés & 696 & 1345 & 2041 \\
mâles & & & \\
femelles & 90 & 1605 & 1695 \\
Total & 35 & 696 & 731 \\
Total & 125 & 2301 & 2426 \\
\cline { 2 - 4 } & 821 & 3646 & 4467 \\
\hline
\end{tabular}


Tableau III. - Liste et effectifs des phlébotomes capturés.

\begin{tabular}{|c|c|c|c|c|c|c|c|c|c|c|c|}
\hline \multirow{3}{*}{$\begin{array}{l}\text { Espèces de } \\
\text { phlébotomes }\end{array}$} & \multicolumn{4}{|c|}{ Troncs déterminés } & \multicolumn{4}{|c|}{ Troncs indéterminés } & \multicolumn{3}{|c|}{ Total général } \\
\hline & \multicolumn{2}{|c|}{ Nancibo } & \multicolumn{2}{|c|}{ F.R.G. } & \multicolumn{2}{|c|}{ Nancibo } & \multicolumn{2}{|c|}{ F.R.G. } & \multirow{2}{*}{ M } & \multirow{2}{*}{$\mathrm{F}$} & \multirow{2}{*}{ Total } \\
\hline & M & $\mathrm{F}$ & M & F & $\mathbf{M}$ & $\mathrm{F}$ & M & F & & & \\
\hline Lu. umbratilis & 285 & 210 & 584 & 315 & 30 & 15 & 1078 & 524 & 1977 & 1064 & 3041 \\
\hline Lu. rorotaensis & 63 & 26 & 259 & 85 & 37 & 13 & 232 & 94 & 591 & 218 & 809 \\
\hline Lu. dendrophyla & 20 & 10 & 24 & 7 & 4 & 0 & 48 & 34 & 96 & 51 & 147 \\
\hline Lu. whitmani & 18 & 0 & 11 & 0 & 0 & 0 & 83 & 26 & 112 & 26 & 138 \\
\hline Lu. abonnenci & 15 & 0 & 20 & 0 & 6 & 0 & 80 & 0 & 121 & 0 & 121 \\
\hline Lu. shannoni & 23 & 2 & 15 & 0 & 8 & 5 & 56 & 2 & 102 & 9 & 111 \\
\hline Lu. spinosa & 10 & 5 & 2 & 1 & 5 & 2 & 5 & l & 22 & 9 & 31 \\
\hline Lu. tuberculata & I & 0 & 4 & 2 & 0 & 0 & 6 & 11 & 11 & 13 & 24 \\
\hline Lu. eliensis & 1 & 0 & 5 & 0 & 0 & 0 & 8 & 0 & 14 & 0 & 14 \\
\hline Lu. ubiquitalis & 1 & 0 & 4 & 2 & 0 & 0 & 2 & 0 & 7 & $?$ & 9 \\
\hline Lu. furcata & 1 & 0 & 0 & 0 & 0 & 0 & 3 & 4 & 4 & 4 & 8 \\
\hline Lu. infraspinosa & 0 & 0 & 0 & 1 & 0 & 0 & 3 & 0 & 3 & 1 & 4 \\
\hline Lu. gomezi & 0 & 1 & 0 & 0 & 0 & 0 & 1 & 0 & 1 & 1 & 2 \\
\hline Lu. punctigeniculata & 1 & 0 & 0 & 0 & 0 & 0 & 0 & 0 & 1 & 0 & 1 \\
\hline Lu. ininii & 1 & 0 & 0 & 0 & 0 & 0 & 0 & 0 & 1 & 0 & 1 \\
\hline Lu. sp. Baduel & 0 & 1 & 0 & 0 & 0 & 0 & 0 & 0 & 0 & 1 & 1 \\
\hline Lu. pilosa & 0 & 0 & 1 & 0 & 0 & 0 & 0 & 0 & 1 & 0 & 1 \\
\hline Lu. aragaoi & 0 & 0 & 1 & 0 & 0 & 0 & 0 & 0 & 1 & 0 & 1 \\
\hline Lu. brachyphalla & 0 & 0 & 1 & 0 & 0 & 0 & 0 & 0 & 1 & 0 & 1 \\
\hline Lut. davisi & 0 & 0 & 1 & $\begin{array}{l}0 \\
0\end{array}$ & 0 & 0 & 0 & 0 & 1 & 0 & 1 \\
\hline Lu. monstruosa & & & 0 & & 0 & & & & 1 & & \\
\hline \multirow{3}{*}{ Total } & 441 & 255 & 932 & 413 & 90 & 35 & 1605 & 696 & \multirow{3}{*}{3068} & \multirow{3}{*}{1399} & \multirow{3}{*}{4467} \\
\hline & \multicolumn{2}{|c|}{696} & \multicolumn{2}{|c|}{1345} & & & \multicolumn{2}{|c|}{2301} & & & \\
\hline & \multicolumn{4}{|c|}{2041} & \multicolumn{4}{|c|}{2426} & & & \\
\hline
\end{tabular}

Deux espèces sont largement dominantes pour les deux stations, Lu. umbratilis et Lu. rorotaensis. Elles représentent $89,7 \%$ à F.R.G. et $83,3 \%$ à Nancibo des phlébotomes capturés. Leur abondance par tronc est 2 fois plus importante pour Lu. umbratilis et 4,5 fois pour Lu. rorotaensis à F.R.G. qu'à Nancibo.

Les phlébotomes récoltés sur troncs indéterminés présentent dans l'ensemble les mêmes densités et répartitions qu'a montré un premier sondage, effectué en avril 1983 à F.R.G. et portant sur 492 phlébotomes et 50 troncs, où Lu. umbratilis et Lu. rorotaensis représentaient $84,3 \%$ des espèces capturées (obs. pers.).

Certaines espèces comme Lu. monstruosa, Lu. punctigeniculata, Lu. ininii, et Lu. sp. de Baduel n'ont été capturées qu'à Nancibo et d'autres comme Lu. infraspinosa, $L u$. aragaoi, Lu. brachyphalla, $L u$. davisi et $L u$. pilosa (nouvellement capturée pour la Guyane) n'ont été prises qu'à F.R.G.

Lu. flaviscutellata et Lu. squamiventris n'ont pas été capturés sur troncs en un an de récolte, alors qu'ils représentent après $L u$. umbratilis les espèces principales capturées sur homme, ce qui montre vraisemblablement qu'ils ont d'autres biotopes que 
les troncs d'arbres. Lu. infraspinosa est également une espèce relativement peu fréquente sur troncs alors qu'elle est capturée sur homme. De même Lu. trichopyga, abondant dans les terriers (Chippaux et al., 1983) n'a pas été retrouvé sur les troncs.

\subsection{Résultats en fonction des essences}

Nous donnons dans le tableau IV la répartition interspécifique des différentes espèces de phlébotomes capturés pour chaque essence et dans le tableau $V$ la distribution intraspécifique des espèces en fonction de chaque essence.

TABleau IV. - Répartition interspécifique des phlébotomes pour chaque essence.

\begin{tabular}{|c|c|c|c|c|c|c|c|c|c|c|}
\hline 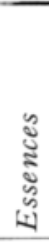 & 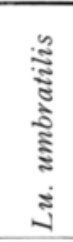 & 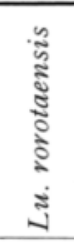 & 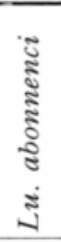 & 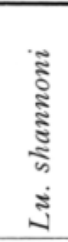 & 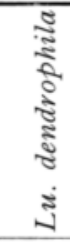 & 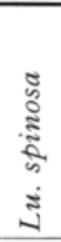 & 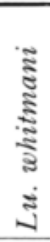 & 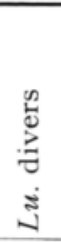 & 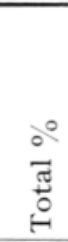 & 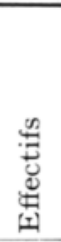 \\
\hline 1 & 48,8 & 42,5 & 1,7 & 2,2 & 2,2 & 0,3 & 1,4 & 0,9 & 100 & 351 \\
\hline 2 & 75,9 & 12,0 & 3,0 & 2,0 & 2,5 & 1,5 & 3,5 & 0,0 & 100 & 199 \\
\hline 3 & 53,7 & 33,3 & 9,3 & 0,0 & 3,7 & 0,0 & 0,0 & 0,0 & 100 & 54 \\
\hline 4 & 76,0 & 18,1 & 1,5 & 2,0 & 1,5 & 0,0 & 0,5 & 0,5 & 100 & 204 \\
\hline 5 & 74,8 & 12,1 & 2,5 & 2,5 & 3,4 & 1,9 & 1,5 & 1,5 & 100 & 206 \\
\hline 6 & 28,3 & 65,0 & 1,7 & 0,0 & 3,3 & 0,0 & 0,0 & 1,7 & 100 & 60 \\
\hline 7 & 74,8 & 14,1 & 0,4 & 1,5 & 4,8 & 0,4 & 1,5 & 2,5 & 100 & 206 \\
\hline 8 & 79,2 & 18,8 & 0,0 & 0,0 & 0,0 & 0,0 & 1,0 & 1,0 & 100 & 101 \\
\hline 9 & 84,0 & 7,5 & 0,0 & 1,1 & 1,1 & 1,1 & 2,1 & 3,2 & 100 & 94 \\
\hline 10 & 75,4 & 19,7 & 0,0 & 0,0 & 1,6 & 0,0 & 0,0 & 3,3 & 100 & 61 \\
\hline 11 & 79,7 & 11,0 & 3,1 & 3,1 & 3,1 & 0,0 & 0,0 & 0,0 & 100 & 64 \\
\hline 12 & 66,7 & 16,7 & 8,3 & 0,0 & 2,8 & 0,0 & 0,0 & 5,6 & 100 & 36 \\
\hline 13 & 31,2 & 53,3 & 1,3 & 7,8 & 1,3 & 1,3 & 2,6 & 1,3 & 100 & 77 \\
\hline 14 & 62,5 & 25,0 & 0,0 & 0,0 & 12,5 & 0,0 & 0,0 & 0,0 & 100 & 8 \\
\hline 15 & 78,3 & 8,7 & 0,0 & 4,4 & 4,4 & 0,0 & 0,0 & 4,4 & 100 & 23 \\
\hline 16 & 58,3 & 0,0 & 4,2 & 12,5 & 8,3 & 4,2 & 8,3 & 4,2 & 100 & 24 \\
\hline 17 & 88,1 & 0,9 & 0,9 & 2,8 & 2,8 & 1,9 & 0,9 & 1,9 & 100 & 109 \\
\hline 18 & 77,5 & 11,3 & 0,0 & 0,0 & 6,9 & 2,5 & 1,3 & 0,6 & 100 & 160 \\
\hline
\end{tabular}

Nous ne retiendrons pour le moment que les espèces bien représentées permettant une interprétation de leurs relations avec la végétation compte tenu des chiffres dont nous disposons.

$L u$. umbratilis fréquente toutes les essences. Nous trouvons pour 11 d'entre elles au moins $75 \%$ de phlébotomes de cette espèce, avec un minimum de $28 \%$ sur le Niamboka (Pouteria guianensis) et un maximum de $88 \%$ sur le Kopi (Goupia glabra).

$L u$. rorotaensis semble plus dépendant de l'essence, tout au moins au niveau 0-1,5 m du sol. En effet, peu d'exemplaires ont été capturés sur certains arbres des essences Balata pomme (Ecclinusa sanguinolenta), Chawari (Caryocar glabrum) et Kopi, sinon aucun sur Akoinsiba (Pouteria sp.). 
Tableau V. - Distribution intraspécifique des phlébotomes pour chaque essence.

\begin{tabular}{|c|c|c|c|c|c|c|c|c|}
\hline \multirow{2}{*}{ 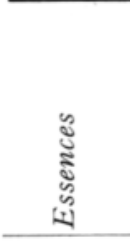 } & 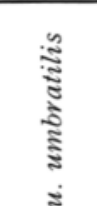 & 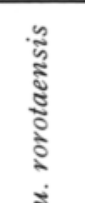 & 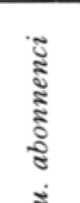 & 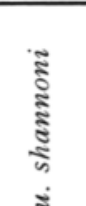 & 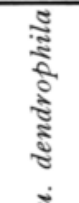 & 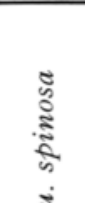 & 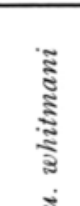 & \multirow{2}{*}{ 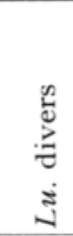 } \\
\hline & 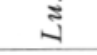 & ت્ & تِ & $\stackrel{\Xi}{3}$ & 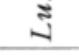 & ذ્ّ & $\stackrel{\Xi}{3}$ & \\
\hline 1 & 3,1 & 10,4 & 4,6 & 5,8 & 3,3 & 1,8 & 5,3 & 2,6 \\
\hline 2 & 5,5 & 2,9 & 3,9 & 5,8 & 4,4 & 8,8 & 15,2 & 0,0 \\
\hline 3 & 3,1 & 7,6 & 23,3 & 0,0 & 5,1 & 0,0 & 0,0 & 0,0 \\
\hline 4 & 16,6 & 15,5 & 14,0 & 17,9 & 7,7 & 0,0 & 6,6 & 5,5 \\
\hline 5 & 5,5 & 3,5 & 7,9 & 7,6 & 5,9 & 11,5 & 6,6 & 5,4 \\
\hline 6 & 1,8 & 16,4 & 4,6 & 0,0 & 5,1 & 0,0 & 0,0 & 5,4 \\
\hline 7 & 5,5 & 4,1 & 1,4 & 4,5 & 8,4 & 2,6 & 6,6 & 9,1 \\
\hline 8 & 8,6 & 8,0 & 0,0 & 0,0 & 0,0 & 0,0 & 6,6 & 5,4 \\
\hline 9 & 8,5 & 2,9 & 0,0 & 4,5 & 2,6 & 8,8 & 13,3 & 16,0 \\
\hline 10 & 4,9 & 5,0 & 0,0 & 0,0 & 2,6 & 0,0 & 0,0 & 10,7 \\
\hline 11 & 5,5 & 2,9 & 9,3 & 9,0 & 5,1 & 0,0 & 0,0 & 0,0 \\
\hline 12 & 2,6 & 2,5 & 14,0 & 0,0 & 2,6 & 0,0 & 0,0 & 10,7 \\
\hline 13 & 1,3 & 8,6 & 2,3 & 13,5 & 1,3 & 4,4 & 6,6 & 2,6 \\
\hline 14 & 0,5 & 0,8 & 0,0 & 0,0 & 2,6 & 0,0 & 0,0 & 0,0 \\
\hline 15 & 1,9 & 0,8 & 0,0 & 4,5 & 2,6 & 0,0 & 0,0 & 5,4 \\
\hline 16 & 1,5 & 0,0 & 4,6 & 13,5 & 5,1 & 8,8 & 13,3 & 5,4 \\
\hline 17 & 10,3 & 0,4 & 4,6 & 13,5 & 7,7 & 17,7 & 6,6 & 10,7 \\
\hline 18 & 13,3 & 7,6 & 0,0 & 0,0 & 28,1 & 35,5 & 13,3 & 5,4 \\
\hline Total \% & 100 & 100 & 100 & 100 & 100 & 100 & 100 & 100 \\
\hline Effectifs & 1394 & 433 & 35 & 40 & 61 & 18 & 29 & 27 \\
\hline
\end{tabular}

Par contre, il a une fréquence élevée sur Niamboka à F.R.G. (65\%) et Dodomissinga (Parkia nitida) (53\%) à Nancibo qui sont des essences où Lu. umbratilis a ses plus faibles valeurs (28 et $31 \%$ ). De plus sa distribution intraspécifique est majoritaire par rapport à $L u$. umbratilis qui ne représente sur ces essences que 1,8 et 1,3\%. L'Angélique et le Taapoutiki (Dendrobangia boliviana) semblent être également d'autres essences propices à la présence de Lu. rorotaensis.

L'association de $L u$. umbratilis et de Lu. rorotaensis pour chaque essence représente au minimum $58 \%$ des phlébotomes capturés.

Certains arbres, correspondant aux essences Taapoukiti, l'association Bioudou (Eperua falcata) - Bois violet, Kopi, Balata pomme, sont plus spécialement fréquentées par l'ensemble des phlébotomes, mais quelques essences semblent héberger plus spécialement les espèces les moins bien représentées.

Signalons le Wacapou (Vouacapoua americana), le Kapokier (Bombax nervosum) et l'Akoinsiba pour Lu. abonnenci, le Taapoutiki et le Dodomissinga pour Lu. shannoni, le Gonfolo-Kouali (Qualea sp.) et le Bougou-bougou pour Lu. dendrophyla, le Kopi, l'association Bioudou - Bois violet et l'Akoinsiba pour Lu. spinosa, enfin l'Angélique et l'Akoinsiba pour Lu. whitmani. 


\section{4- Conclusion}

L'importance de la végétation dans la biologie des phlébotomes en région intertropicale a déjà été mise en lumière.

En Amazonie, Ready et coll., (1983 et 1984) ont relevé le développement préférentiel de certaines espèces dans des associations phyto-écologiques ou phyto-topographiques données : Lu flaviscutellata dans les forêts non climaciques tant primaires que secondaires et Psychodopygus wellcomei, vecteur probable de $L$. braziliensis, dans les forêts de moyenne altitude.

L'étude des phénomènes à une échelle plus fine a montré l'influence de la structure forestière dans la dispersion des espèces phlébotomiennes. Ainsi les troncs à contreforts, étudiés par Christensen en 1982 à Panama, se sont avérés être d'excellents gîtes de repos pour certains vecteurs de Leishmania braziliensis comme Lu. ylephiletor, $L u$. trapidoi ou d'autres espèces non anthropophiles comme $L u$. shannoni. Toujours à Panama, Chaniotis a montré que $L u$. rorotaensis et Lu. micropyga se situaient préférentiellement près de la canopée. A l'inverse, nous avons trouvé en forêt guyanaise une population non négligeable de $L u$.rorotaensis à la base des troncs.

Le Pont et coll. (1980) ont signalé (en Guyane française) que certaines essences à fûts réguliers comme l'Angélique étaient beaucoup plus fréquentées que d'autres troncs découpés comme le Tabebuia par exemple.

L'objectif de notre travail a résidé dans une étude plus poussée de la composition des associations floristiques, susceptible de mettre en évidence des phénomènes ayant échappé à des études plus globales.

Il en ressort assez nettement que des relations privilégiées existent entre essences végétales et populations de phlébotomes. Certaines essences semblent être plus propices au développement ou au maintien des espèces de forêt guyanaise, soit comme gîtes d'attente ou de repos, soit comme support au développement larvaire. Toutefois, l'existence de nombreux paramètres difficilement mesurables limite actuellement l'analyse des causes de ce phénomène.

La poursuite de ces recherches en relation avec l'étude des différents facteurs écologiques devrait permettre une meilleure connaissance des relations phlébotomes/ végétation.

Remerciements. Les auteurs remercient les personnels de l'Office National des Forêts de Guyane française pour leur aide efficace sur le terrain, et tout particulièrement MM. M. Gazel, ingénieur des Eaux et Forêts, G. Evrard, technicien forestier, B. Guard et D. Machine, agents techniques.

\section{BIBLIOGRAPHIE}

Chaniotis B. N., Tesh R. B., Correa M. A., Johnson K. M. : Diurnal resting sites of Phlebotomine sand flies in a panamanian tropical forest. $J$. Med. Ent., 1972, 9, 91-98.

Chippaux J. P., PAJOT F. X. : La Leishmaniose en Guyane française. 4. Note préliminaire sur les Phlébotomes de terriers. Cah. O.R.S.T.O.M., ser. Ent. méd. Parasitol., I983, 21, 149-I 54. 
Christensen H. A., Vasguez A. de M. : The tree-buttress biotope : a pathobiocenose of Leihmania braziliensis. Am. J. Trop. Med. Hyg., 1982, 3I, 243-25I.

Dedet J. P., Pajot F. X., DesjeuX P., Goyot P., ChippauX J. P., Geoffroy B. : Natural hosts of Leishmania mexicana amazonensis (Lainson, Shaw, I972) (Kinetoplastidae, Trypanosomatidae) in French Guiana. Trans. Roy. Soc. Trop. Med. Hyg., 1985a, 79. 302-305.

Dedet J. P., Pradinaud R., Desjeux P., Jacquet-Vialet P., Girardeau I., Esterre P., Gotz W. : Deux premiers cas de leishmaniose cutanée à Leishmania mexicana amazonensis en Guyane française. Bull. Soc. Path. Exot., 1985b, 78, 64-7o.

Floch H., Abonnenc E. : Faune de l'Union Française. XIV. Diptère. Phlébotomes de la Guyane et des Antilles françaises. Faune tropicale, $n^{\circ}$ XIV, O.R.S.T.O.M., Paris, 1952, 212 p.

Foratrini O. P. : Entomologia medica, $4^{e}$ volume. Psychodidae, Phlebotominae, Leishmaniose, Bartonellose. E. Blücher Ed., Univ. São Paulo, 1973, 658 p.

Martins A. V., Williams P., Falcao A. L. : American sand flies (Diptera : Psychodidae, Phlebo. tominae). Acad. Brasil. de Cièncias, Rio de Janeiro, 1978, $195 \mathrm{p}$.

LE Pont F., PAjot F. X. : La Leishmaniose en Guyane française $\mathrm{r}$. Étude de l'écologie et du taux d'infection naturelle du vecteur Lutzomyia (Nyssomyia) umbratilis en saison sèche. Considérations épidémiologiques. Cah. O.R.S.T.O.M., sér. Ent. méd. Parasitol., I980, I8, 359-382.

READY P. D. LAINSon R., Shaw J. J. : Leishmaniasis in Brazil XX. Prevalence of " enzootic rodent leishmaniasis " (Leishmania mexicana amazonensis), and apparent absence of "pian bois " (Le. braziliensis guyanensis), in plantations of introduced tree species and in other non climax forests in eastern Amazonia. Trans. Roy. Soc. Trop. Med. Hyg., 1983, 77, 775-785.

Ready P. D., Lainson R., Shaw J. J. : Habitat and seasonality of Psychodopygus wellcomen help incriminate it as a vector of Leishmania braziliensis in Amazônia and Northeast Brazil. Trans. Roy. Soc. Trop. Med. Hyg., 1984, 78, 543-544. 